\title{
Lab-on-a-disc platform for screening of genetically modified E. coli cells via cell-free electrochemical detection of $p$-Coumaric acid
}

Sanger, Kuldeep; Zor, Kinga; Jendresen, Christian Bille; Heiskanen, Arto; Amato, Letizia; Nielsen, Alex Toftgaard; Boisen, Anja

Published in:

Sensors and Actuators B: Chemical

Link to article, DOI:

10.1016/j.snb.2017.06.184

Publication date:

2017

Document Version

Peer reviewed version

Link back to DTU Orbit

Citation (APA):

Sanger, K., Zor, K., Jendresen, C. B., Heiskanen, A., Amato, L., Nielsen, A. T., \& Boisen, A. (2017). Lab-on-adisc platform for screening of genetically modified $E$. coli cells via cell-free electrochemical detection of $p$ -

Coumaric acid. Sensors and Actuators B: Chemical, 253, 999-1005. https://doi.org/10.1016/j.snb.2017.06.184

\section{General rights}

Copyright and moral rights for the publications made accessible in the public portal are retained by the authors and/or other copyright owners and it is a condition of accessing publications that users recognise and abide by the legal requirements associated with these rights.

- Users may download and print one copy of any publication from the public portal for the purpose of private study or research.

- You may not further distribute the material or use it for any profit-making activity or commercial gain

- You may freely distribute the URL identifying the publication in the public portal 


\title{
Lab-on-a-disc platform for screening of genetically modified $E$. coli cells via cell-free electrochemical detection of $p$-Coumaric acid
}

Kuldeep Sanger ${ }^{a^{*}}$, Kinga Zór ${ }^{a^{*}}$, Christian Bille Jendresen ${ }^{b}$, Arto Heiskanen ${ }^{a}$, Letizia Amato ${ }^{a}$, Alex Toftgaard Nielsen $^{\mathrm{b}}$, Anja Boisen $^{\mathrm{a}}$

${ }^{a}$ Department of Micro- and Nanotechnology, Technical University of Denmark, 2800 Kgs. Lyngby, DENMARK

${ }^{\mathrm{b}}$ The Novo Nordisk Foundation Center for Biosustainability, Technical University of Denmark, 2970 Hørsholm, DENMARK

*Corresponding authors:

Kuldeep Sanger, Technical University of Denmark, Department of Micro-and Nanotechnology, Ørsteds Plads, Building 345C, room 116, 2800 Kgs. Lyngby, Denmark, Tel: +454525634, email: sakuld@ nanotech.dtu.dk

Kinga Zór, Technical University of Denmark, Department of Micro-and Nanotechnology, Ørsteds Plads, Building 345C, room 101E, 2800 Kgs. Lyngby, Denmark, Tel:+4545255751, email:kinzo@ nanotech.dtu.dk

\begin{abstract}
We present a robust easy to use lab-on-a-disc (LoD) device with integrated sample pre-treatment and electrochemical detection system for cell-free detection of a secondary metabolite, $p$-Coumaric acid (pHCA), produced by genetically modified E. coli. In the LoD device, which incorporates eight filtration and electrochemical detection units, the sample filtration was performed by rotating the disc using a programmable closed-loop stepper motor. The electrodes, patterned on plastic substrate, were connected through a printed circuit board to the slip ring using a robust magnetic clamping system that enables easy assembly and robust electrical connections. pHCA was quantified in a linear range from 0.125 up to $2 \mathrm{mM}$ using square wave voltammetry. The platform was successfully used for the quantification of pHCA produced by two genetically modified $E$. coli strains after $24 \mathrm{~h}$ of cell culture. The data obtained from the electrochemical measurements showed good correlation with high performance liquid chromatographic analysis. The developed LoD system offers fast and easy detection of pHCA, enabling screening of genetically modified organisms based on the quantity of produced secondary metabolites.
\end{abstract}

Keywords: lab-on-a-disc; cell free detection; electrochemical sensor; bacterial metabolite; magnetic clamping; electrode on plastic substrate 


\section{Introduction}

In the past few years, the interest for plant secondary metabolites has increased significantly due to their diverse biological functions and wide range of applications in chemical, food, health, cosmetic, and pharmaceutical industries (Balasundram et al., 2006; Barbulova et al., 2015; Soto et al., 2015; Sytar Oksana, 2012). $p$-Coumaric acid (pHCA) is one of these secondary metabolites with many physiological effects, acting as, e.g., a potent antioxidant, antimicrobial, anxiolytic, analgesic, antimutagen, sedative and immunoregulatory agent (Boz, 2015; Cheng et al., 2007; Ou et al., 2012). Unfortunately, extraction from plants requires a significant amount of starting material, harsh conditions and results in a limited purity of products (Ou et al., 2012). To overcome these challenges, scientists have altered the genome of microorganisms to develop metabolically engineered microbial strains for a more economical production of pHCA from inexpensive amino acids (Calero et al., 2016; Rodriguez et al., 2015; Xue et al., 2014). Biosynthesis of pHCA can be carried out via deamination of tyrosine (Tyr) by incorporating tyrosine ammonia lyase (TAL) gene in E. coli genome (Jendresen et al., 2015). Efficient optimization of the tyrosine pathway as well as the activity of the TAL enzyme for production of pHCA is generally validated by the screening of the genetically modified strains by quantifying the amount of produced pHCA. High performance liquid chromatography (HPLC), gas chromatography, thin-layer chromatography and spectrophotometric assays are commonly used to quantify secondary metabolites from plants (Khoddami et al., 2013). However, there is a great need for novel platforms that allow high-throughput analysis with minimal sample handling for cost-effective, easy and fast screening of modified strains.

Centrifugal microfluidic devices, often referred to as lab-on-a-disc (LoD) systems, have gained interest in both academic research and industry (Burger et al., 2016; Smith et al., 2016). In a LoD platform, the centrifugal forces, induced by rotating the device, enable the transport and manipulation of liquids; therefore offer an alternative to traditional pressure-driven microfluidic systems. They require minimal instrumentation for liquid transport and handling, consequently enabling the development of a simple, compact and integrated detection systems. Although optical detection is the most commonly implemented approach on LoD devices (Donolato et al., 2015; Grumann et al., 2006; Kim et al., 2013; Koh et al., 2015; Uddin et al., 2016; Zhang et al., 2015), there are examples describing the integration of electrochemical (Abi-Samra et al., 2013; Amato et al., 2015; Andreasen et al., 2015; Cho et al., 1982; Kim et al., 2013; Li et al., 2013; Nwankire et al., 2015; Rattanarat et al., 2015) and mechanical (Bache et al.,2013; Bosco et al., 2011) sensors. Integration of electrochemical detection with microfluidic devices offers advantages, such as possibility to miniaturize both the transducer (electrode) and instrumentation as well as multiplexing and automation of measurements without losing the performance (Nyholm, 2005; Sassa et al., 2008; Xu et al., 2009). In addition, electrochemical detection is affected by neither sample turbidity nor optical path length, and it is possible to fabricate low-cost detection systems with minimal power demand (Felipe et al., 2014; Xu et al., 2011). However, direct detection of analytes in a complex sample matrix can be challenging when the potential interfering compounds and the possibility for surface fouling of the transducer are taken into 
consideration. There are several reports on the integration of various sample handling (e.g. mixing, dilution) and pre-treatment methods (e.g. DNA extraction, blood separation, cell extraction from plasma) for LoD based applications (Grumann et al., 2006; Kim et al., 2014; Li et al., 2013; Nwankire et al., 2015) to improve detection accuracy.

In this work, we have developed a simple, robust and easy to use LoD platform with eight electrode arrays fabricated on a plastic substrate to facilitate electrochemical detection of pHCA in supernatants derived from E. coli cultures. To provide cell-free samples to the electrochemical detection units the LoD device had an integrated microporous filter membrane for simple sample pre-treatment. The platform was used for quantification of pHCA produced by two genetically modified $E$. coli strains.

\section{Material and methods}

\subsection{Chemicals}

Potassium hexacyanoferrate(II) (ferrocyanide), potassium hexacyanoferrate(III) (ferricyanide), potassium hydroxide $(\mathrm{KOH})$, phosphate buffered saline (PBS), pHCA, the components of the culture medium for E. coli (minimal M9 medium) and the analytes used for the HPLC analysis were purchased from Sigma-Aldrich ${ }^{\circledR}$ Co. (St. Louis, MO, USA) unless otherwise stated. Minimal M9 medium, containing $6.8 \mathrm{~g} / \mathrm{L} \mathrm{Na}_{2} \mathrm{HPO}_{4} \cdot 7 \mathrm{H}_{2} \mathrm{O}, 3.0 \mathrm{~g} / \mathrm{L} \mathrm{KH}_{2} \mathrm{PO}_{4}, 0.5 \mathrm{~g} / \mathrm{L} \mathrm{NaCl}, 1.0 \mathrm{~g} / \mathrm{L} \mathrm{NH}_{4} \mathrm{Cl}, 2 \mathrm{mM} \mathrm{MgSO}{ }_{4}, 0.1$ $\mathrm{mM} \mathrm{CaCl}_{2}$, and 1:2000 (v/v) dilution of a trace element stock solution $\left(10 \mathrm{~g} / \mathrm{L} \mathrm{FeCl} \mathrm{Fe}_{3} \cdot 6 \mathrm{H}_{2} \mathrm{O}, 2 \mathrm{~g} / \mathrm{L}\right.$ $\mathrm{ZnSO}_{4} \cdot 7 \mathrm{H}_{2} \mathrm{O}, 0.4 \mathrm{~g} / \mathrm{L} \mathrm{CuCl}{ }_{2} \cdot 2 \mathrm{H}_{2} \mathrm{O}, 1 \mathrm{~g} / \mathrm{L} \mathrm{MnSO}{ }_{4} \cdot \mathrm{H}_{2} \mathrm{O}, 0.6 \mathrm{~g} / \mathrm{L} \mathrm{CoCl} \cdot 6 \mathrm{H}_{2} \mathrm{O}$, and $3.2 \mathrm{~mL} / \mathrm{L}$ of $0.5 \mathrm{M}$ EDTA at $\mathrm{pH} 8$ ), was prepared in water and autoclaved. $10 \mathrm{~g} / \mathrm{L}$ glucose, $1 \mathrm{mM}$ isopropyl $\beta$-D-1thiogalactopyranoside (IPTG), and Wolfe's Vitamin solution (purchased from ATCC® (LGC Standards, UK) and containing $10 \mu \mathrm{g} / \mathrm{L}$ pyridoxine- $\mathrm{HCl}, 5 \mu \mathrm{g} / \mathrm{L}$ thiamine- $\mathrm{HCl}, 5 \mu \mathrm{g} / \mathrm{L}$ riboflavin, 5 $\mu \mathrm{g} / \mathrm{L}$ nicotinic acid, $5 \mu \mathrm{g} / \mathrm{L}$ calcium D-(+)-pantothenate, $5 \mu \mathrm{g} / \mathrm{L} p$-aminobenzoic acid, $2 \mu \mathrm{g} / \mathrm{L}$ biotin, 2 $\mu \mathrm{g} / \mathrm{L}$ folic acid, $5 \mu \mathrm{g} / \mathrm{L}$ lipoic acid, and $0.1 \mu \mathrm{g} / \mathrm{L}$ vitamin B12) were added to the autoclaved medium. In addition $2 \mathrm{mM}$ Tyr was added as a substrate for pHCA production during cell culture. To maintain the plasmids in the developed E. coli strains, antibiotics, chloramphenicol and spectinomycin, were also added to the M9 medium at the final concentration of $34 \mathrm{mg} / \mathrm{L}$ and $50 \mathrm{mg} / \mathrm{L}$, respectively and. M9 buffer was prepared using $6.8 \mathrm{~g} / \mathrm{L} \mathrm{Na} 2 \mathrm{HPO} 4 \cdot 7 \mathrm{H} 2 \mathrm{O}, 3.0 \mathrm{~g} / \mathrm{L} \mathrm{KH} 2 \mathrm{PO} 4$, and $0.5 \mathrm{~g} / \mathrm{L} \mathrm{NaCl}$. Ethanol (EtOH) 99\% was purchased from VWR Chemicals (Søborg, Denmark). All the aqueous solutions were prepared in ultrapure water obtained from a Milli-Q ${ }^{\circledR}$ water purification system (Millipore Corporation, Billerica, MA, USA).

\subsection{E. coli culture}

E. coli control (CBJ786) and pHCA producing strains (CBJ792 and CBJ800) are derived as previously described (Jendresen et al., 2015) from the expression strain BL21(DE3)pLysS (Invitrogen/Life Technologies), carrying either the extrachromosomal plasmid pCDFDuet-1 (CBJ786) or derivative plasmids encoding for one of the tyrosine ammonia-lyases R_XAL (CBJ792) or FjTAL (CBJ800). For pHCA production assays, the $E$. coli strains were grown in $180 \mathrm{~mL}$ of $\mathrm{M} 9$ medium in $500 \mathrm{~mL}$-flasks in 
a temperature-controlled orbital shaker $\left(30{ }^{\circ} \mathrm{C}, 250 \mathrm{rpm}\right) .30 \mathrm{~mL}$ samples were withdrawn at $24 \mathrm{~h}$, and supernatants were obtained by centrifugation $\left(10 \mathrm{~min}\right.$ at $\left.10000 \mathrm{~g}, 4{ }^{\circ} \mathrm{C}\right)$. The optical density (OD) at $600 \mathrm{~nm}$ was measured in cuvettes with $1 \mathrm{~cm}$ path length using a UV-1600PC spectrophotometer (VWR, Radnor, Pennsylvania, USA).

\subsection{Experimental setup}

The fully assembled experimental set-up shown in Figure 1A is comprised of the LoD device mounted on the spin-stand, which consists of an electronic triggering system for the high-resolution camera (pco.pixelfly, PCO AG, Kelheim, Germany) and a spinning motor (Maxon motor, model RE 35) controlled by custom-made software in a LabVIEW environment. The electrodes (Figure 2B) incorporated in the fluidic platform were connected to a CHI1030A potentiostat ( $\mathrm{CH}$ Instruments, Inc., Austin, TX, USA ) through a custom-made high-speed electrical slip ring with operating speed range of 0-2500 rpm and dynamic resistance $<0.01 \Omega$ (Model: SRS110073, Penlink, Sweden). The potentiostat was connected to the stationary part of the slip ring, whereas the moving part was coupled to the custom-made printed circuit board (PCB). The gold plated spring-loaded pins (Mill-Max Mfg. Corp., Oyster Bay, NY, USA) soldered to the PCB, formed the connection to the electrodes patterned on the disc (Figure 1B). The wires soldered to the PCB (Figure 1B, inset) enabled the connection to the potentiostat via the slip ring (Figure 1A).

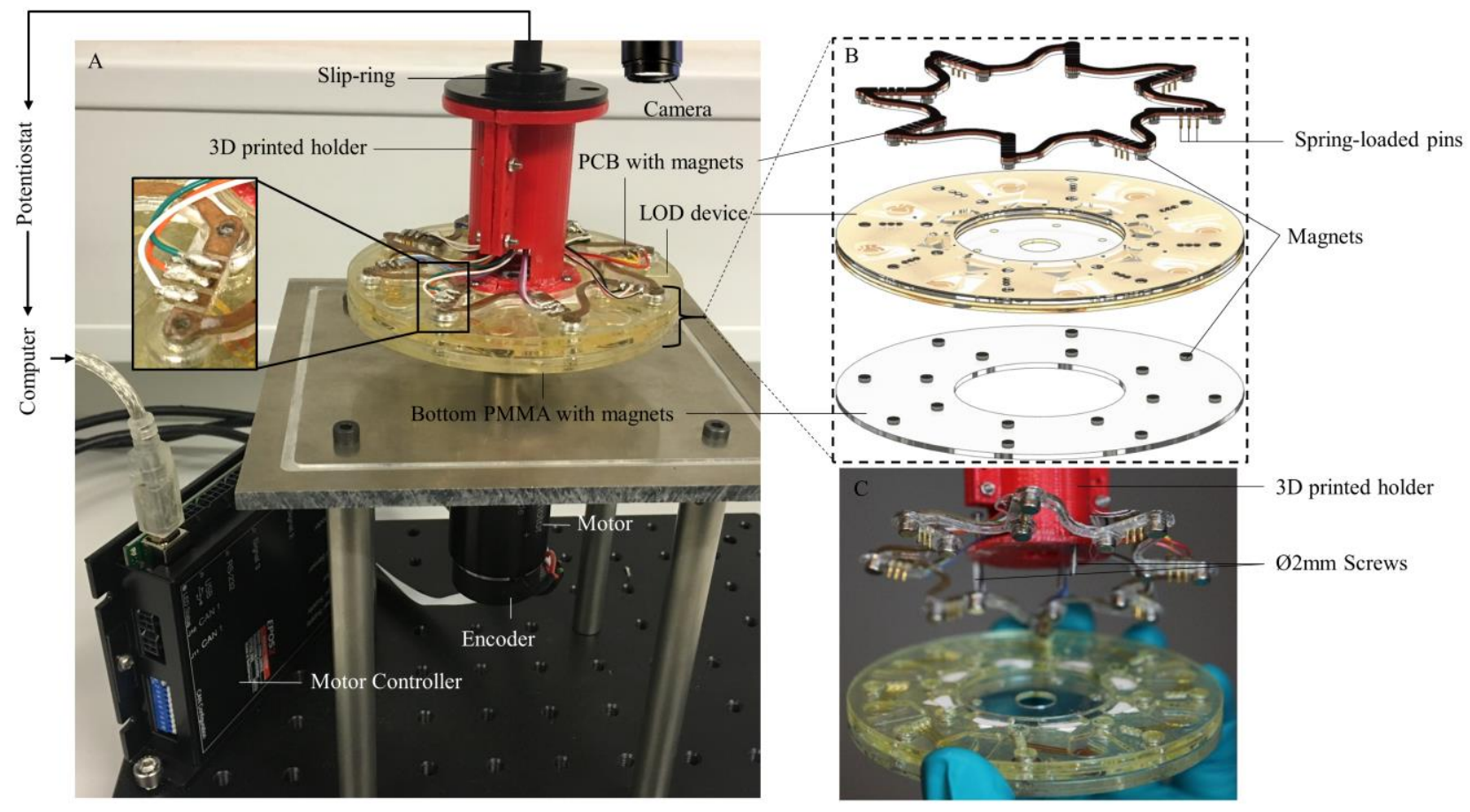

Figure 1. (A) Photograph of a fully assembled experimental setup (slip ring connection to spring-loaded pins through PCB in the inset). (B) Exploded schematic view of the magnetic clamping system. (C) Photograph of the microfluidic disc and PCB with the magnetic clamping. 
As can be seen in Figure 1B, the easy coupling of the PCB to the LoD device was achieved by incorporating rod magnets of $\varnothing 4 \mathrm{~mm}$ and $3 \mathrm{~mm}$ height (Webcraft $\mathrm{GmbH}$, Gottmadingen, Germany) in the poly(methyl methacrylate) (PMMA) layer $(1 \mathrm{~mm})$ glued to the PCB and in an additional PMMA layer $(2 \mathrm{~mm}$ ) placed below the LoD (Figure 1B). The slip ring was held in place by a 3D printed (Printer: MakerBot Industries, LLC, Brooklyn, NY, USA) holder made of polylactic acid (Reprap Aps, Copenhagen, Denmark), which was fixed on top of the disc mounted on the spin-stand using Ø 2-mm screws (Figure1A and 1C).

\subsection{Design, fabrication and assembly of microfluidic LoD device}

The parts of the LoD device were designed using the computer aided design (CAD) software SolidWorks (Dassault Systèmes SolidWorks Corporation, Waltham, MA, USA). Based on the CAD files, the optical grade PMMA (Evonik Industries AG, Darmstadt, Germany) and double-sided pressure sensitive adhesive (PSA) tape (140 $\mu \mathrm{m}$ thick, ARcare ${ }^{\circledR} 90106$ from Adhesive Research Ltd., Limerick, Ireland) were cut using a computer-controlled Epilog Mini 18 laser cutter equipped with a $30 \mathrm{~W} \mathrm{CO}_{2}$ laser (Epilog Laser, Golden, CO, USA) and Cameo 3 blade cutter (Silhouette, Inc., Lindon, UT, USA), respectively. The whole LoD assembly comprised four PMMA sheets, which were bonded to each other using a PSA, tape used interspersed between 4 layers of PMMA sheets (Figure 2A).
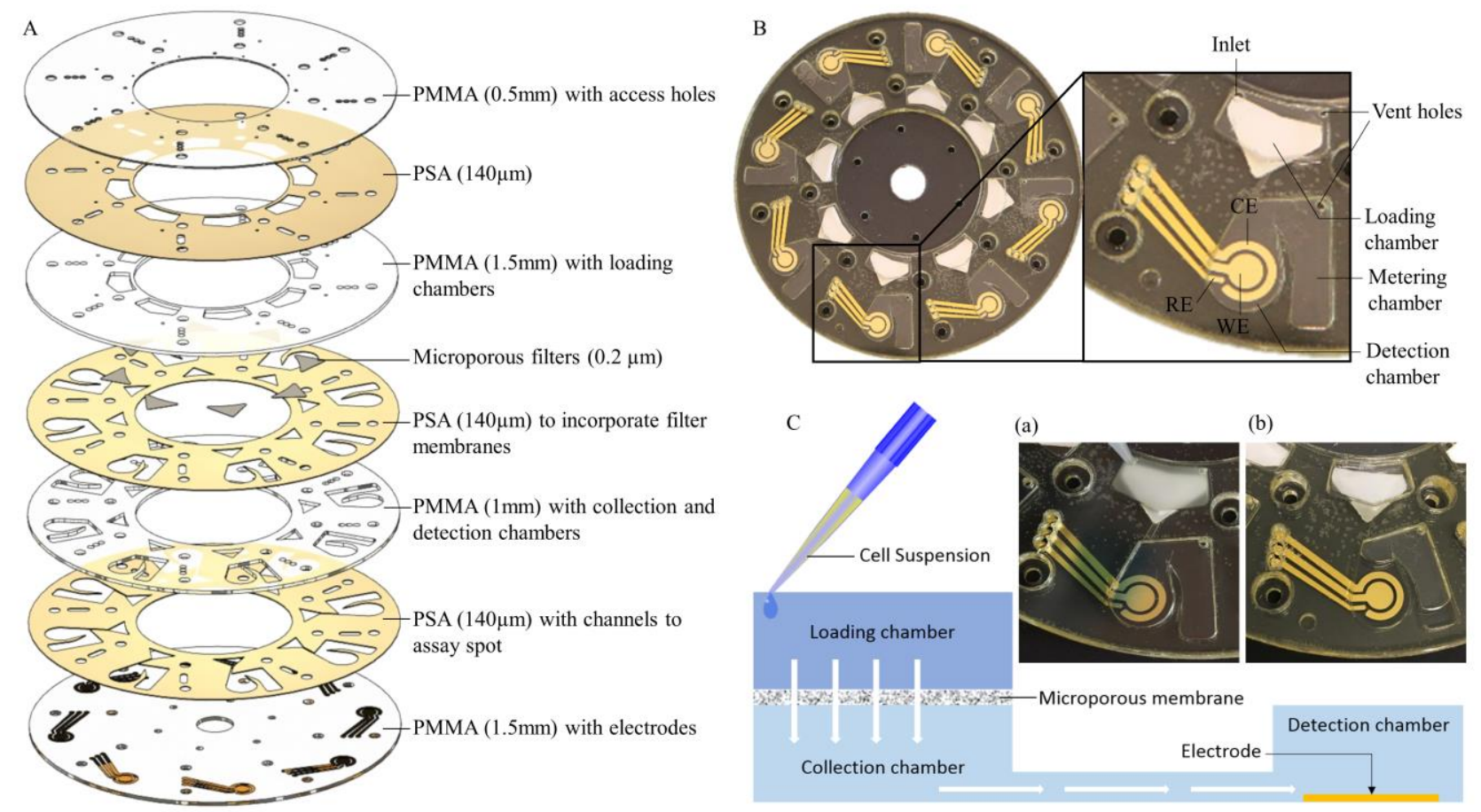

Figure 2. (A) Exploded view of the microfluidic disc with electrodes patterned on the bottom PMMA sheet. (B) Photograph of the microfluidic disc (details of a filtration and detection unit with electrode array in the inset). (C) Schematics of the cross section of the microfluidic layout (the arrows show the flow direction during rotation): sample (a) before and (b) after on-disc filtration. 
The second PSA layer also allowed incorporation of the microporous cellulose acetate membranes (Tisch Scientific, North Bend, OH, USA) with $0.2 \mu \mathrm{m}$ pore size.

The filter membranes were custom cut using the blade cutter to fit the top of the collection chamber and placed between the PMMA sheets to separate the loading chambers from the collection chambers (Figure 2A, C). The turbid cell suspension was loaded into the loading chamber (Figure 2C(a)) and the clear cell-free suspension was driven to the detection chamber (Figure $2 \mathrm{C}(\mathrm{b})$ ) after filtration achieved by rotating the LoD device at $700 \mathrm{rpm}$ for 5 minutes. The PMMA sheet incorporated the eight gold electrode arrays (Figure 2B) each comprising of a working (WE), counter (CE), and pseudo-reference electrode (RE). The electrodes were patterned on the bottom PMMA substrate of the disc assembly using e-beam evaporation (20 nm Cr adhesion layer/200 nm Au) through a steel shadow mask, fabricated with the laser micromachining instrument as described previously (Amato et al., 2015).

\subsection{Electrochemical measurements}

All the electrochemical measurements were performed at room temperature with an eight-channel potentiostat (CHI1030A). For electrode characterization, cyclic voltammograms (CV) were recorded from $-400 \mathrm{mV}$ to $400 \mathrm{mV}$ vs. Au pseudo-RE in $10 \mathrm{mM}$ ferri/ferrocyanide using PBS (pH 7.4) as supporting electrolyte. Cleaning of the electrodes was carried out in $50 \mathrm{mM} \mathrm{KOH}$ dissolved in ultrapure water, with potential sweep from $-200 \mathrm{mV}$ to $-1200 \mathrm{mV}$ vs. Au pseudo-RE (scan rate $50 \mathrm{mV}$ $\mathrm{s}^{-1}$ ), performing at least three sweeps. pHCA detection was performed using square wave voltammetry (SWV) with a step potential of $0.001 \mathrm{~V}$, square-wave frequency of $10 \mathrm{~Hz}$ and amplitude of $0.05 \mathrm{~V}$. The applied potential ranges were 150-800 mV (control (J786) supernatant), 100-600 mV (M9 buffer), and 100-700 mV (M9 bacterial culture medium). The slip ring facilitates measurements during rotation as demonstrated by Andreasen et al. (Andreasen et al., 2015) yet, the electrochemical measurements presented in this paper were performed under static condition.

\subsection{HPLC analysis}

pHCA detection in $5 \mu \mathrm{L}$ injection volumes was performed using a Waltham HPLC system (Thermo Scientific, MA, USA) equipped with a UV detector $(333 \mathrm{~nm})$ as previously described (Jendresen et al., 2015). The separation was achieved in a Discovery HS F5 column ( $3 \mu \mathrm{m}$ particle size, $15 \mathrm{~cm} \times 4.6$

$\mathrm{mm}$ ) at $30^{\circ} \mathrm{C}$ under gradient elution with a two-solvent system (A: $10 \mathrm{mM}$ ammonium formate (pH 3.0 adjusted with formic acid) and $\mathrm{B}$ : acetonitrile) at a flow rate of $0.7 \mathrm{~mL} / \mathrm{min}$. The elution started at $5 \% \mathrm{~B}$ followed by linear increase of the fraction to $60 \%$ between 1.5 and $7 \mathrm{~min}$ after injection. The fraction of B was decreased back to $5 \%$ between 9.5 and 9.6 min, remaining there until 12 min.

\subsection{Data analysis}

For the calibration curves, the peak heights were obtained from the original data files using the CHI data analysis software and plotted against the corresponding concentration using OriginPro 2015 (OriginLab Corporation Northampton, MA, USA). The presented SWVs were background-subtracted 
and baseline-corrected to improve the visualization and identification of peaks over the baseline without introducing any artifacts.

\section{Results and Discussion}

\subsection{Electrochemical characterization and re-usability of the on-disc electrodes}

Metal electrodes are most commonly fabricated on silicon or glass substrates although there are reports describing electrode fabrication on plastic substrates aiming at creating low-cost (Xu et al., 2011) and easy to integrate electrodes for microfluidic applications (Matteucci et al., 2016; Šnita et al., 2009). Patterning electrodes on plastic substrates can be achieved in a cost-efficient way by screen printing (Metters et al., 2011) or by using a stencil or shadow mask in combination with e-beam evaporation (Amato et al., 2015; Xu et al., 2011). However, it may be challenging to fabricate electrodes capable of providing a reproducible electrochemical signal as well as a stable metal (e.g. gold) layer, which is crucial for sensor development. The performance and reproducibility of the electrodes fabricated on PMMA substrate were evaluated by acquiring CVs. As shown in Figure 3A, the eight electrodes on the same disc have a peak potential separation $\left(\Delta \mathrm{E}_{\mathrm{p}}\right)$ of $98 \pm 2 \mathrm{mV}$ and the ratio between the reduction and oxidation peaks was $1.07 \pm 0.01$. In addition, a linear relation between the oxidation and reduction peak currents and the square root of the scan rates was observed as presented in Figure $3 \mathrm{~B}$ for sixteen electrodes from two discs. The recorded CVs indicate a quasi-reversible behavior of the redox couple on the electrodes and an excellent electrochemical reproducibility of the electrodes on the same disc as well as between discs. Although the observed $\Delta \mathrm{E}_{\mathrm{p}}$ was greater than $59 \mathrm{mV}$, which is the theoretically expected value for a reversible single-electron transfer reaction such that of ferri/ferrocyanide (A.J. Bard, 2001), the values are comparable with those reported for gold electrodes fabricated on glass (Andreasen et al., 2015), plastic (Matteucci et al., 2016) and silicon substrates (Zor et al., 2014).
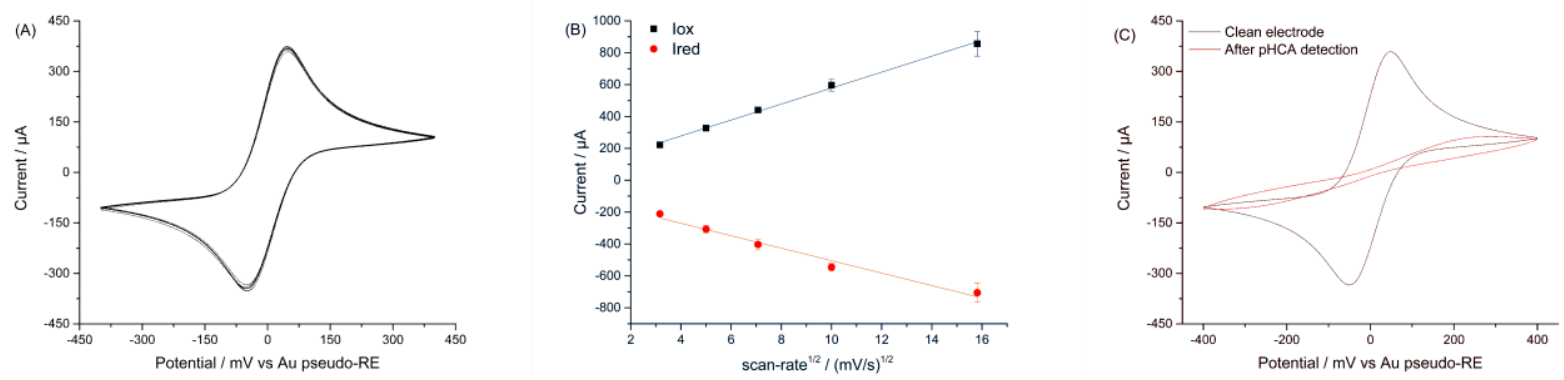

Figure 3. (A) CVs for 8 electrodes on the same disc acquired in $10 \mathrm{mM}$ ferri/ferrocyanide using $\mathrm{PBS}$ (pH 7.4) as supporting electrolyte (scan rate $50 \mathrm{mVs}^{-1}$ ). (B) Oxidation and reduction peak currents as a function of the square root of the scan rate $\left(10-250 \mathrm{mVs}^{-1}\right)$. The error bars represent the standard deviation of 16 electrodes among two discs (8 electrodes per disc). (C) Representative CVs for one- electrode acquired in $10 \mathrm{mM}$ ferri/ferrocyanide before and after $\mathrm{pHCA}$ detection (scan rate $50 \mathrm{mVs}^{-1}$ ). 
Electrochemical oxidation of pHCA leads to the formation of a polymeric film on the electrode, impeding diffusion of the analyte and its oxidation on the electrode surface (Janeiro et al., 2007). In order to be able to re-use the electrodes after pHCA detection, cleaning and regeneration of the electrodes were evaluated. As shown in Figure 3C, the oxidation and reduction peaks of ferri/ferrocyanide are not clearly detectable after pHCA detection. There are various physical and chemical methods available for cleaning gold surfaces (Fischer et al., 2009). Due to the fact that the electrodes were fabricated on the plastic substrate, the optimal electrode cleaning process proposed by Fisher et al. (Fischer et al., 2009) proved to be unsuitable due to electrode delamination. However, the electrode surface could be cleaned by linear sweep voltammetry in $50 \mathrm{mM} \mathrm{KOH}$ solution in the potential range between -200 and $-1200 \mathrm{mV}$ (vs. on disc Au pseudo RE) at the scan rate of $50 \mathrm{mV} / \mathrm{s}$ as previously shown by Zor et al. (Zor et al., 2014). When CVs recorded for sixteen clean electrodes on two discs were compared with electrodes cleaned after pHCA detection we observed a slight increase in $\Delta \mathrm{E}_{\mathrm{p}}$ (from $98 \pm 2$ to $111 \pm 9 \mathrm{mV}$ ) as well as decrease in the oxidation and reduction peak currents (from $421 \pm 6$ to $398 \pm 11 \mu \mathrm{A}$ and $-392 \pm 7$ to $-361 \pm 16 \mu \mathrm{A}$, respectively). The increase in $\Delta \mathrm{E}_{\mathrm{p}}$ and decrease in the oxidation and reduction peak currents indicated that the electrochemical performance of the electrodes was not completely regained after pHCA detection. However, due to the good on-disc electrode reproducibility, the slight decrease in electrode performance can be overcome by performing on-disc calibration prior to determining the pHCA content in real samples.

\section{2 pHCA quantification in supernatant}

The electrochemical oxidation of pHCA was thoroughly investigated by Janeiro et al. using various electrochemical techniques (Janeiro et al., 2007). SWV was used in this work for quantification of pHCA because it is widely used in the development of electrochemical sensors, given its sensitivity and reliability (Chen and Shah, 2013). Detecting pHCA in cell culture supernatant could be a challenge due to the presence of possible interfering compounds that may be electrochemically active at the same potential as the target analyte. As a first step in the development of the detection method, pHCA was determined in M9 buffer (Figure S1A) and M9 culture medium (Figure S1B). However, our goal was to determine pHCA directly in bacterial supernatants, therefore we evaluated pHCA detection in a sample matrix that is very similar to the real samples, namely supernatant from the control (non pHCA producing) E. coli strain (CBJ786) collected after $24 \mathrm{~h}$ culture. The control strain has similar growth kinetics as CBJ792 and CBJ800 strains, but does not produce pHCA (Figure S2A). Signals recorded for $500 \mu \mathrm{M}$ pHCA added to M9 buffer, M9 medium and to the bacterial culture supernatant of the control (CBJ786) are shown in Figure S1A, B, C. The observed difference in the pHCA oxidation potential is probably due to the $\mathrm{pH}$ dependent electrochemical oxidation of pHCA (Janeiro et al., 2007) since the $\mathrm{pH}$ of the supernatant at $24 \mathrm{~h}$ is close to $\mathrm{pH} 5.8$, whereas the $\mathrm{pH}$ of $\mathrm{M} 9$ medium is 6.8 , and of the M9 buffer is 7.4. We found that there are no electrochemical interferences in the SWV recorded between 150 and $800 \mathrm{mV}$ in the range where pHCA is detected in control supernatant and therefore it was further used for the construction of calibration curves. Figure 4A shows representative SWVs for pHCA in the concentration range from $125 \mu \mathrm{M}$ to $3 \mathrm{mM}$ recorded in spiked control bacterial 
supernatant. Figure 4B shows the linear part of the calibration curve. The pHCA concentrations in supernatants of recombinant microorganisms commonly reach $0.5-10 \mathrm{mM}$ under laboratory conditions (Jendresen et al., 2015; Rodriguez et al., 2015; Xue et al., 2014). Therefore, the sensor array seems to be suitable for determining the analyte either directly or after dilution.
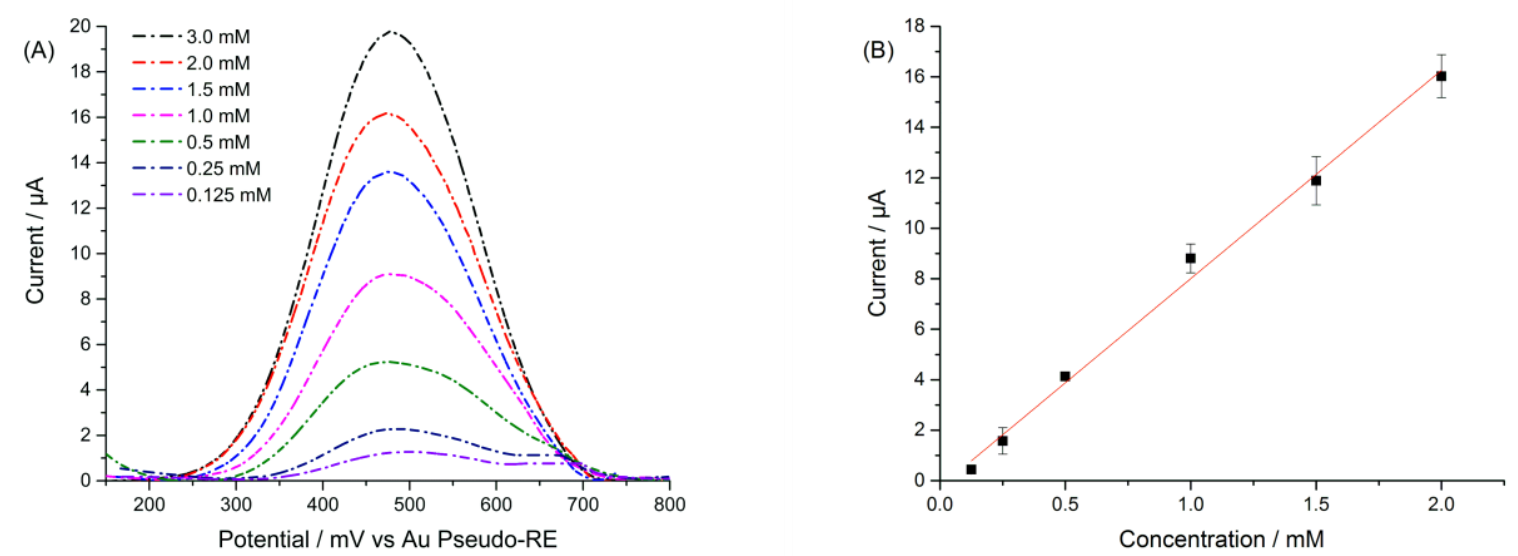

Figure 4. (A) SWVs of increasing pHCA concentration in control bacterial supernatant, potential step $0.001 \mathrm{~V}$, frequency $10 \mathrm{~Hz}$, amplitude $0.05 \mathrm{~V}$. (B) Linear part of pHCA calibration curve recorded from spiked control bacterial supernatant, the error bars represent the standard deviation, $n=4$.

\subsection{On-disc cell filtration and pHCA detection from real samples}

Electrochemical sensors are prone to fouling (Hanssen et al., 2016). Therefore, the effect of the presence of bacterial cells on pHCA detection was investigated. In a typical bacterial culture, the cell density, determined as $\mathrm{OD}$ at $600 \mathrm{~nm}$ wavelength, can change from less than 0.2 to more than 10 (S2A). When determining pHCA in the presence of cell density, corresponding to OD600 of 2.0 after $9 \mathrm{~h}$ of growth, the recorded signal was not influenced by the presence of cells (S3). However, in a $24 \mathrm{~h}$ cell culture with high cell density, the electrochemical signal was 30 to $50 \%$ lower due to the sedimentation of cells on the electrode that caused electrode fouling (Figure 5). Since we aimed at determining the pHCA produced at the end point of the bacterial culture $(24 \mathrm{~h})$, a cell filtration unit $(0.2$ $\mu \mathrm{m}$ pore size) was integrated on the LoD platform as described in the Materials and methods section 2.4. The on-disc filtration was carried out after filling the loading chamber with $120 \mu \mathrm{l}$ cell suspension followed by spinning the disc at $700 \mathrm{rpm}$ for 5 minutes. The centrifugal forces push the filtered cell suspension to the detection chamber incorporated with the electrode at the bottom as it is shown in Figure $2 \mathrm{C}$. The detection chamber was specifically designed by connecting it to a metering chamber as depicted in Figure $2 \mathrm{~B}$ to enable the filling of the detection chamber with a defined volume which is independent of the filtration rate.

CBJ792 and CBJ800 E. coli strains, carrying derivative plasmids encoding TAL and the CBJ786 control strain were grown for $24 \mathrm{~h}$ in M9 medium supplemented with $2 \mathrm{mM}$ Tyr. The pHCA production efficiencies of the genetically modified strains depend on their ability to convert Tyr into 
pHCA as they express different recombinant TALs (Jendresen et al., 2015). After $24 \mathrm{~h}$, almost the entire amount of Tyr was metabolized and or converted into pHCA (S2B, S2C). Therefore, Tyr did not interfere with the electrochemical detection of pHCA.

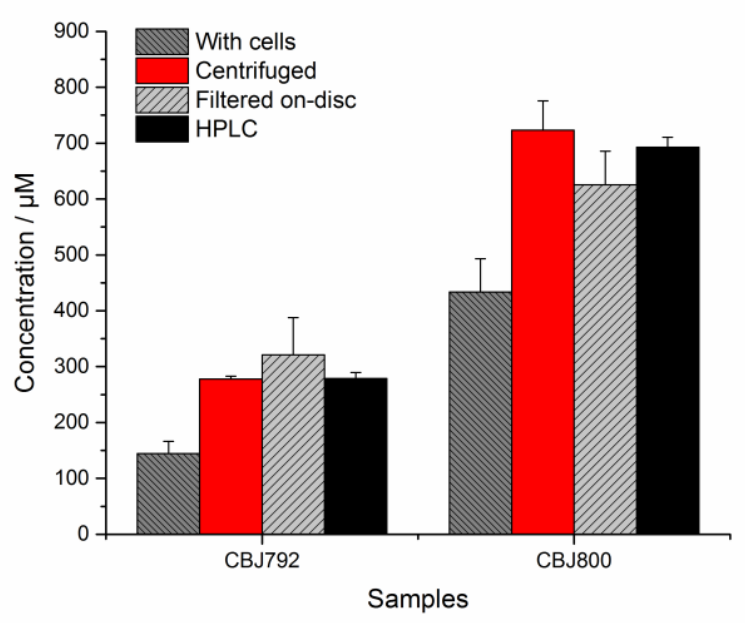

Figure 5. pHCA quantification in presence of cells, on-disc filtered and centrifuged supernatant vs. HPLC, the error bar represents the standard deviation between measurements, $n=3$.

The pHCA content in the genetically modified E. coli cultures were measured at the end of bacterial growth at $24 \mathrm{~h}$ in bacterial culture supernatant. Cells were removed by on-disc filtration and the electrochemical detection was carried out under static condition after the detection chamber was filled. The pHCA concentration was calculated based on the peak current values of the recorded SWVs using the calibration curve. The electrochemically determined pHCA content in centrifuged and on-disc filtered samples was found to correlate well with the data obtained from HPLC analysis (Figure 5).

\section{Conclusion}

Monitoring of biological processes is important when developing new genetically modified strains or evaluating the quality of an already well-known process. In this work, we used pHCA producing $E$. coli as a model system for developing a LoD device for bioprocess monitoring. Due to the lack of natural availability of and the demand for plant secondary metabolites in the manufacturing of healthcare and nutritional products, microbial organisms are often engineered to contain artificial biosynthetic pathways. We presented a robust, easy to use and compact electrochemical LoD device with integrated filtration for detection of pHCA produced by genetically modified E. coli strains as an alternative approach to traditionally used methods for pHCA quantification. The gold electrodes fabricated on plastic (PMMA) substrate proved to be stable and reusable as well as facilitates reproducible measurements. The electrochemical quantification of the pHCA content at the end of the bacterial culture measured for two $E$. coli strains was in a good agreement with values obtained from HPLC 
analysis. The presented device can be further developed for time-dependent monitoring of pHCA content by including additional sample pre-treatment steps to enable pHCA detection in the presence of high Tyr concentration. In addition, by further parallelization to facilitate higher detection throughput, the sensing platform has a great potential for being implemented as a compact analytical tool for bioprocess monitoring.

\section{Acknowledgements}

This work was financially supported by the European Research Council under the European Union's Seventh Framework Programme (FP7/2007-2013) grant no. 320535-HERMES. The authors also acknowledge the support from the Novo Nordisk Foundation (grant no. NNF15OC0015246) and from the IDUN project (grant no. DNRF122) funded by the Danish National Research Foundation and the Velux Foundations. The authors acknowledge Robert Burger for useful discussions related to the development of the fluidic system.

\section{References}

A.J. Bard, L.R.F. (Ed.), 2001. Electrochemical Methods: Fundamentals and Applications, 2001. second ed., John Wiley and Sons, Hoboken, NJ,.

Abi-Samra, K., Kim, T.-H., Park, D.-K., Kim, N., Kim, J., Kim, H., Cho, Y.-K., Madou, M., 2013. Electrochemical velocimetry on centrifugal microfluidic platforms. Lab Chip 13, 3253. doi:10.1039/c3lc50472g

Amato, L., Sanger, K., Tehrani, S.E., Burger, R., Caviglia, C., Andreasen, Z., Heiskanen, A., Emnéus, J., Boisen, A., 2015. A lab-on-a-disc platform for trapping of cells, monitoring of cell behaviour and evaluation of redox metabolism, in: Proceedings of MicroTAS 2015. Chemical and Biological Microsystems Society, pp. 1299-1302.

Andreasen, S.Z., Kwasny, D., Amato, L., Brogger, A.L., Bosco, F.G., Andersen, K.B., Svendsen, W.E., Boisen, A., 2015. Integrating Electrochemical Detection with Centrifugal Microfluidics for RealTime and Fully Automated Sample Testing. RSC Adv 5, 17187-17193. doi:10.1039/C4RA16858E

Bache, M., Bosco, F.G., Brøgger, A.L., Frøhling, K.B., Alstrøm, T.S., Hwu, E.-T., Chen, C.-H., EugenOlsen, J., Hwang, I.-S., Boisen, A., 2013. Nanomechanical recognition of prognostic biomarker suPAR with DVD-ROM optical technology. Nanotechnology 24, 444011. doi:10.1088/0957$4484 / 24 / 44 / 444011$

Balasundram, N., Sundram, K., Samman, S., 2006. Phenolic compounds in plants and agri-industrial by-products: Antioxidant activity, occurrence, and potential uses. Food Chem. 99, 191-203. doi:10.1016/j.foodchem.2005.07.042 
Barbulova, A., Colucci, G., Apone, F., 2015. New Trends in Cosmetics: By-Products of Plant Origin and Their Potential Use as Cosmetic Active Ingredients. Cosmetics 2, 82-92.

doi:10.3390/cosmetics2020082

Bosco, F.G., Hwu, E.-T., Chen, C.-H., Keller, S., Bache, M., Jakobsen, M.H., Hwang, I.-S., Boisen, A., 2011. High throughput label-free platform for statistical bio-molecular sensing. Lab Chip 11, 2411-6. doi:10.1039/c1lc20116f

Boz, H., 2015. p-Coumaric acid in cereals: Presence, antioxidant and antimicrobial effects. Int. J. Food Sci. Technol. 50, 2323-2328. doi:10.1111/ijfs.12898

Burger, R., Amato, L., Boisen, A., 2016. Detection methods for centrifugal microfluidic platforms. Biosens. Bioelectron. 76, 54-67. doi:10.1016/j.bios.2015.06.075

Calero, P., Jensen, S.I., Nielsen, A.T., 2016. Broad host range ProUSER vectors enable fast characterization of inducible promoters and optimization of p-coumaric acid production in Pseudomonas putida KT2440. ACS Synth. Biol. doi:10.1021/acssynbio.6b00081

Chen, A., Shah, B., 2013. Analytical Methods Electrochemical sensing and biosensing based on square wave voltammetry. Anal. Lett. 2158-2173. doi:10.1039/c3ay40155c

Cheng, J.C., Dai, F., Zhou, B., Yang, L., Liu, Z.L., 2007. Antioxidant activity of hydroxycinnamic acid derivatives in human low density lipoprotein: Mechanism and structure-activity relationship. Food Chem. 104, 132-139. doi:10.1016/j.foodchem.2006.11.012

Cho, H.K., Lee, Y.H., Couch, R.A., Jagadeesh, J.M., Olson, C.L., 1982. Development of a multichannel electrochemical centrifugal analyzer. Clin. Chem. 28, 1956-1961.

Donolato, M., Antunes, P., Bejhed, R.S., Zardán Gómez De La Torre, T., Østerberg, F.W., Strömberg, M., Nilsson, M., Strømme, M., Svedlindh, P., Hansen, M.F., Vavassori, P., 2015. Novel readout method for molecular diagnostic assays based on optical measurements of magnetic nanobead dynamics. Anal. Chem. 87, 1622-1629. doi:10.1021/ac503191v

Felipe, A., Cruz, D., Norena, N., Kaushik, A., Bhansali, S., 2014. Biosensors and Bioelectronics A low-cost miniaturized potentiostat for point-of-care diagnosis. Biosens. Bioelectron. 62, 249-254. doi:10.1016/j.bios.2014.06.053

Fischer, L.M., Tenje, M., Heiskanen, A.R., Masuda, N., Castillo, J., Bentien, A., Émneus, J., Jakobsen, M.H., Boisen, A., 2009. Microelectronic Engineering Gold cleaning methods for electrochemical detection applications. Microelectron. Eng. 86, 1282-1285. doi:10.1016/j.mee.2008.11.045

Grumann, M., Steigert, J., Riegger, L., Moser, I., Enderle, B., Riebeseel, K., Urban, G., Zengerle, R., Ducrée, J., 2006. Sensitivity enhancement for colorimetric glucose assays on whole blood by onchip beam-guidance. Biomed. Microdevices 8, 209-214. doi:10.1007/s10544-006-8172-x

Hanssen, L.B., Shajahan, S., Wong, K.Y.D., 2016. Recent strategies to minimise fouling in electrochemical detection systems. Rev Anal Chem 35, 1-28.

Janeiro, P., Novak, I., Seruga, M., Oliveira-Brett, A.M., 2007. Electroanalytical Oxidation of $p$ Coumaric Acid. Anal. Lett. 40, 3309-3321. doi:10.1080/00032710701672822 
Jendresen, C.B., Stahlhut, S.G., Li, M., Gaspar, P., Siedler, S., Förster, J., Maury, J., Borodina, I., Nielsen, A.T., 2015. Highly Active and Specific Tyrosine Ammonia-Lyases from Diverse Origins Enable Enhanced Production of Aromatic Compounds in Bacteria and Saccharomyces cerevisiae. Appl. Environ. Microbiol. 81. doi:10.1128/AEM.00405-15

Khoddami, A., Wilkes, M. a., Roberts, T.H., 2013. Techniques for analysis of plant phenolic compounds. Molecules 18, 2328-2375. doi:10.3390/molecules18022328

Kim, T.-H., Park, J., Kim, C.-J., Cho, Y.-K., 2014. Fully integrated lab-on-a-disc for nucleic acid analysis of food-borne pathogens. Anal. Chem. 86, 3841-8. doi:10.1021/ac403971h

Kim, T., Abi-samra, K., Sunkara, V., Park, D., Amasia, M., Kim, N., Kim, J., Kim, H., Madou, M., Cho, Y.-K., 2013. Flow-enhanced electrochemical immunosensors on centrifugal microfluidic platforms. Lab Chip 3747-3754. doi:10.1039/c31c50374g

Koh, C.Y., Schaff, U.Y., Piccini, M.E., Stanker, L.H., Cheng, L.W., Ravichandran, E., Singh, B.R., Sommer, G.J., Singh, A.K., 2015. Centrifugal microfluidic platform for ultrasensitive detection of botulinum toxin. Anal. Chem. 87, 922-928. doi:10.1021/ac504054u

Li, T., Fan, Y., Cheng, Y., Yang, J., 2013. An electrochemical Lab-on-a-CD system for parallel whole blood analysis. Lab Chip 13, 2634-40. doi:10.1039/c31c00020f

Matteucci, M., Heiskanen, A., Zór, K., Emnéus, J., Taboryski, R., 2016. Comparison of Ultrasonic Welding and Thermal Bonding for the Integration of Thin Film Metal Electrodes in Injection Molded Polymeric Lab-on-Chip Systems for Electrochemistry. Sensors (Basel). 1795. doi:10.3390/s16111795

Metters, J.P., Kadara, R.O., Banks, C.E., 2011. New directions in screen printed electroanalytical sensors: an overview of recent developments. Analyst 136, 1067-1076. doi:10.1039/c0an00894j

Nwankire, C.E., Venkatanarayanan, A., Glennon, T., Keyes, T.E., Forster, R.J., Ducrée, J., 2015. Label-free impedance detection of cancer cells from whole blood on an integrated centrifugal microfluidic platform. Biosens. Bioelectron. 68, 382-389. doi:10.1016/j.bios.2014.12.049

Nyholm, L., 2005. Electrochemical techniques for lab-on-a-chip applications. Analyst 599-605. doi: $10.1039 / \mathrm{b} 415004 \mathrm{j}$

Ou, S.Y., Teng, J.W., Zhao, Y.Y., Zhao, J., 2012. p -coumaric Acid Production from Lignocelluloses. Phenolic Acids Compos. Appl. Heal. Benefits.

Rattanarat, P., Teengam, P., Siangproh, W., Ishimatsu, R., Nakano, K., Chailapakul, O., Imato, T., 2015. An Electrochemical Compact Disk-type Microfluidics Platform for Use as an Enzymatic Biosensor. Electroanalysis 27, 703-712. doi:10.1002/elan.201400590

Rodriguez, A., Kildegaard, K.R., Li, M., Borodina, I., Nielsen, J., 2015. Establishment of a yeast platform strain for production of p-coumaric acid through metabolic engineering of aromatic amino acid biosynthesis. Metab. Eng. 31, 181?188. doi:10.1016/j.ymben.2015.08.003

Sassa, F., Morimoto, K., Satoh, W., Suzuki, H., 2008. Electrochemical techniques for microfluidic. Electrophoresis 1787-1800. doi:10.1002/elps.200700581 
Smith, S., Mager, D., Perebikovsky, A., Shamloo, E., Kinahan, D., Mishra, R., Torres Delgado, S.M., Kido, H., Saha, S., Ducrée, J., Madou, M., Land, K., Korvink, J.G., 2016. CD-based microfluidics for primary care in extreme point-of-care settings. Micromachines 7, 1-32. doi:10.3390/mi7020022

Šnita, D., Schrott, W., Svoboda, M., 2009. Microelectronic Engineering Metal electrodes in plastic microfluidic systems. Microelectron. Eng. 86, 1340-1342. doi:10.1016/j.mee.2009.01.001

Soto, M., Falqué, E., Domínguez, H., 2015. Relevance of Natural Phenolics from Grape and Derivative Products in the Formulation of Cosmetics. Cosmetics 2, 259-276. doi:10.3390/cosmetics2030259

Sytar Oksana, 2012. Plant phenolic compounds for food, pharmaceutical and cosmetics production. J. Med. Plants Res. 6, 2526-2539. doi:10.5897/JMPR11.1695

Uddin, R., Burger, R., Donolato, M., Fock, J., Creagh, M., Fougt, M., Boisen, A., 2016. Lab-on-a-disc agglutination assay for protein detection by optomagnetic readout and optical imaging using nanoand micro- sized magnetic beads. Biosens. Bioelectron. 85, 351-357. doi:10.1016/j.bios.2016.05.023

Xu, X., Zhang, S., Chen, H., Kong, J., 2009. Integration of electrochemistry in micro-total analysis systems for biochemical assays : Recent developments. Talanta 80, 8-18. doi:10.1016/j.talanta.2009.06.039

Xu, Y., Yung, L.H.K., Lee, Y.X.T.M., 2011. Low cost fabrication of microelectrodes on plastic substrate. Microsyst. Technol. 361-366. doi:10.1007/s00542-011-1278-y

Xue, Y., Zhang, Y., Cheng, D., Daddy, S., He, Q., 2014. Genetically engineering Synechocystis sp. Pasteur Culture Collection 6803 for the sustainable production of the plant secondary metabolite p-coumaric acid. Proc. Natl. Acad. Sci. U. S. A. 111, 1-6. doi:10.1073/pnas.1323725111

Zhang, L., Li, X., Li, Y., Shi, X., Yu, H.Z., 2015. Indirect competitive assays on DVD for direct multiplex detection of drugs of abuse in oral fluids. Anal. Chem. 87, 1896-1902. doi:10.1021/ac5040715

Zor, K., Heiskanen, A., Caviglia, C., Vergani, M., Landini, E., Shah, F., Carminati, M., MartínezSerrano, A., Moreno, T.R., Kokaia, M., Benayahu, D., Keresztes, Z., Papkovsky, D., Wollenberger, U., Svendsen, W.E., Dimaki, M., Ferrari, G., Raiteri, R., Sampietro, M., Dufva, M., Emnéus, J., 2014. A compact multifunctional microfluidic platform for exploring cellular dynamics in real-time using electrochemical detection. RSC Adv. 63761-63771. doi:10.1039/C4RA12632G 


\section{Figure captions:}

Figure 1. (A) Photograph of a fully assembled experimental setup (slip ring connection to spring-loaded pins through PCB in the inset). (B) Exploded schematic view of the magnetic clamping system. (C) Photograph of the microfluidic disc and PCB with the magnetic clamping.

Figure 2. (A) Exploded view of the microfluidic disc with electrodes patterned on the bottom PMMA sheet. (B) Photograph of the microfluidic disc (details of a filtration and detection unit with electrode array in the inset). (C) Schematics of the cross section of the microfluidic layout (the arrows show the flow direction during rotation): sample (a) before and (b) after on-disc filtration.

Figure 3. (A) CVs for 8 electrodes on the same disc acquired in $10 \mathrm{mM}$ ferri/ferrocyanide using PBS (pH 7.4) as supporting electrolyte ( $\mathrm{scan}$ rate $50 \mathrm{mVs}^{-1}$ ). (B) Oxidation and reduction peak currents as a function of the square root of the scan rate $\left(10-250 \mathrm{mVs}^{-1}\right)$. The error bars represent the standard deviation of 16 electrodes among two discs (8 electrodes per disc). (C) Representative CVs for one- electrode acquired in $10 \mathrm{mM}$ ferri/ferrocyanide before and after pHCA detection (scan rate $50 \mathrm{mVs}^{-1}$ ).

Figure 4. (A) SWVs of increasing pHCA concentration in control bacterial supernatant, potential step $0.001 \mathrm{~V}$, frequency $10 \mathrm{~Hz}$, amplitude $0.05 \mathrm{~V}$. (B) Linear part of pHCA calibration curve recorded from spiked control bacterial supernatant, the error bars represents the standard deviation, $\mathrm{n}=4$.

Figure 5. pHCA quantification in presence of cells, on-disc filtered and centrifuged supernatant vs. HPLC, the error bar represents the standard deviation between measurements, $\mathrm{n}=3$. 


\section{Supplementary material}

\section{Lab-on-a-disc platform for screening of genetically modified $E$. coli cells via cell-free electrochemical detection of $p$-Coumaric acid}

Kuldeep Sanger $^{\mathrm{a}^{*}}$, Kinga Zór ${ }^{\mathrm{a}^{*}}$, Christian Bille Jendresen ${ }^{\mathrm{b}}$, Arto Heiskanen ${ }^{\mathrm{a}}$, Letizia Amato ${ }^{\mathrm{a}}$, Alex Toftgaard Nielsen $^{\mathrm{b}}$, Anja Boisen ${ }^{\mathrm{a}}$

${ }^{a}$ Department of Micro- and Nanotechnology, Technical University of Denmark, 2800 Kgs. Lyngby, DENMARK ${ }^{\mathrm{b}}$ The Novo Nordisk Foundation Center for Biosustainability, Technical University of Denmark, 2970 Hørsholm, DENMARK
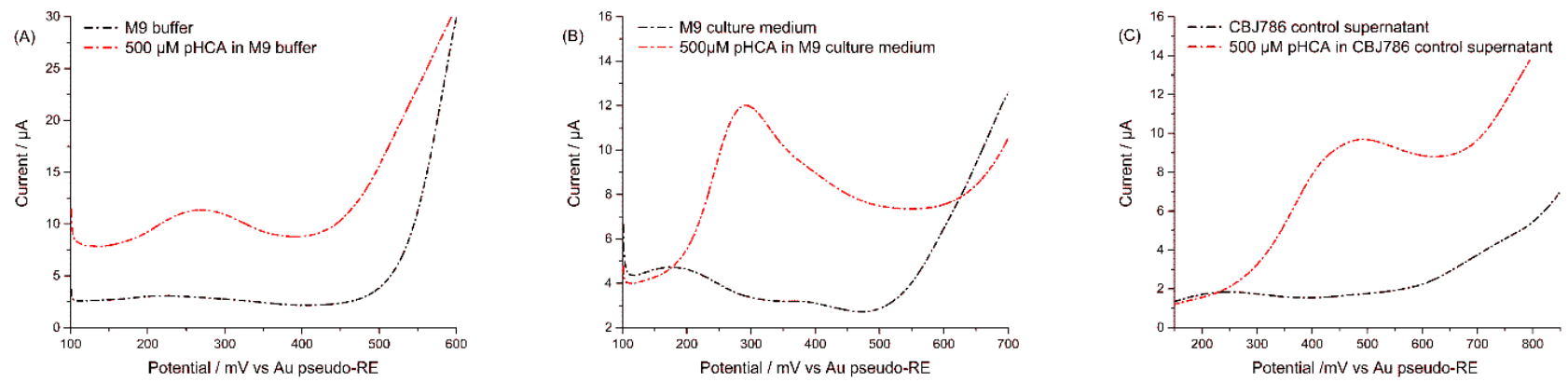

S1. SWVs for $500 \mu \mathrm{M}$ pHCA in M9 buffer (a), M9 culture medium (b) and CBJ786 control supernatant (c) with respective blanks.
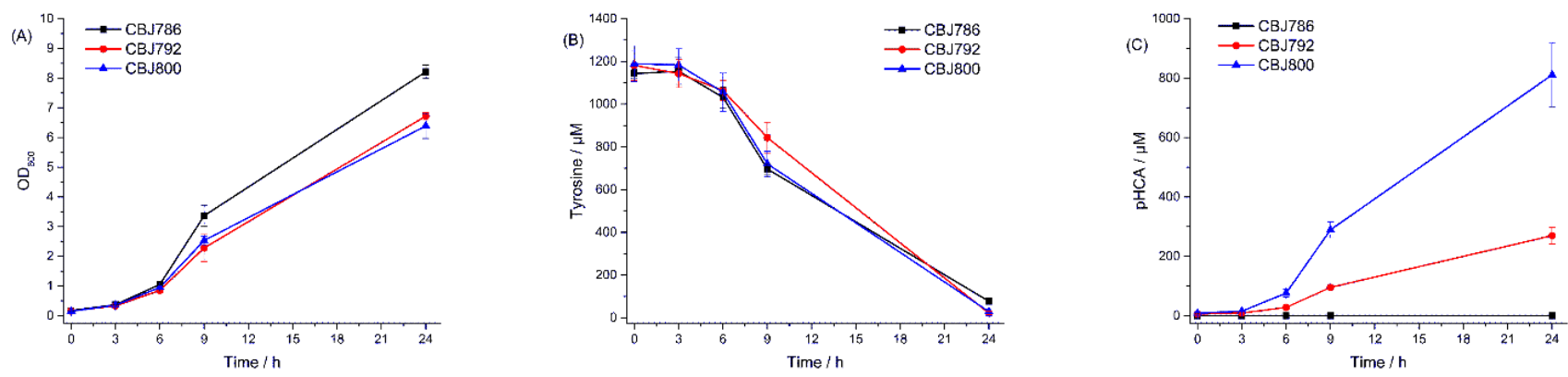

S2. Changes in three different E. coli stains during 24h of culture: Growth (a); Tyr consumption (b) and pHCA production (c). 


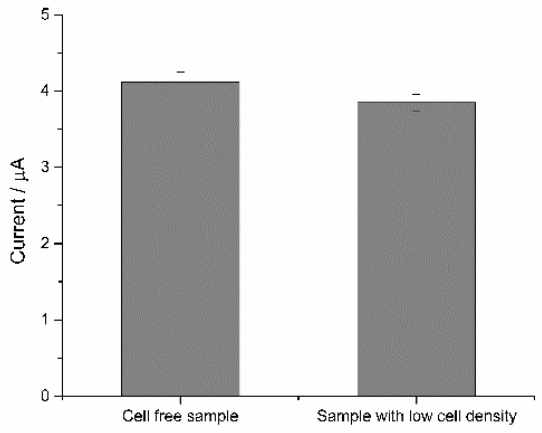

S3. Comparison between signal recorded for $500 \mu \mathrm{M}$ pHCA in the presence $(\mathrm{OD}=2)$ and absence of cells. 